\title{
Locational Marginal Pricing of Natural Gas subject to Engineering Constraints
}

\author{
Aleksandr M. Rudkevich \\ Newton Energy Group \\ Tabors Caramanis Rudkevich \\ Boston, $M A$ \\ arudkevich@negll.com
}

\author{
Anatoly Zlotnik \\ Theoretical Division \\ Los Alamos National Laboratory \\ Los Alamos, NM \\ azlotnik@lanl.gov
}

\begin{abstract}
We derive a price formation mechanism to maximize social welfare for a pipeline network that delivers natural gas from suppliers to consumers. The system is modeled as a metric graph subject to physical balance laws for steadystate hydraulic flow on edges and mass balance at nodes. The pricing mechanism incorporates engineering constraints on local pressures and energy applied by gas compressors. Optimality conditions yield expressions for locational marginal prices for gas ( $g L M P s$ ) and a decomposition of gLMPs into components corresponding to energy, compression, and two types of congestion. We demonstrate that price and pressure differentials between nodes have the opposite sign, so that price cannot decline in the direction of flow, and prove that the pricing mechanism is revenue adequate. We also present computational examples of congestion pricing for a small test network and a large-scale case study.
\end{abstract}

\section{INTRODUCTION}

Significant growth in the use of natural gas for power generation in the United States is causing growing demand for transportation of gas through large-scale interstate pipelines [1]. This trend has been driven by environmental regulations, a push towards cleaner electric power sources, abundant supplies of inexpensive natural gas, and improvements in gas turbine efficiency [2]. Retiring coal-fired and nuclear power plants continue to be replaced primarily by gas-fired units [3]. Because power production by gas turbines is highly maneuverable, gas-fired generators are best able to balance fluctuations caused by variable and non-dispatched generation such as wind and solar [4]. Concurrently, increased reliance on gas-fired generation transfers the energy demand onto natural gas pipeline infrastructure [5], [6].

Current market structures for interstate pipeline transportation services in the United States are constrained within a regulatory framework that was not designed for responsive price formation [7]. Access to pipeline capacity is provided at rates regulated by the Federal Energy Regulatory Commission (FERC). Holders of firm physical rights are allowed to sell unneeded capacity on a daily basis through a release mechanism. Released capacity is bundled with gas supply and traded bilaterally in a locational spot market for natural gas. Trading platforms such as the Intercontinental Exchange (ICE) serve as major vehicles for price formation. Reported price indices for several dozen locations in North America change daily with Friday prices prevailing over the weekend. These daily prices fail to reflect intra-day demand variations.

Historically, intra-day demand variations were primarily caused by changes in residential and commercial loads. These changes are typically weather driven, predictable, and reasonably well managed by pipeline operators. In contrast, significant intra-day and even sub-hourly swings in demand for natural gas as a fuel for electric generation create new challenges for pipeline operators, and pose reliability risks for gas pipelines and electric systems. The need to better coordinate the two sectors to mitigate these risks is well recognized [8], [3], [9], and is reflected in two recent FERC orders [10], [11]. Coordination mechanisms proposed to date are based on widening the scope of operational information exchanged by the two sectors and on adjusting the timing of when these exchanges occur. While these measures are helpful, it is also necessary to create economic tools for gas-electric coordination that provide incentives for market participants to change behavior in a way that would result in more efficient and reliable operation of both infrastructures. Intra-day locational prices of natural gas that are consistent with the physics and engineering constraints of pipeline operation could provide such a tool.

In the electric power industry, the use of optimization to price electricity based on the physical ability of the electric network to deliver it from producers to consumers is standard practice [12], [13]. In contrast, with the exception of the market in the Australian province of Victoria [14], the use of physics-based optimization to clear natural gas markets remains a topic of research [15], [16]. Developing locational pricing mechanisms for natural gas is challenging because of complex physical and engineering factors of pipeline hydraulic modeling and optimization [17], [18]. Thus in addition to the different physical and operational aspects of gas pipelines and electric power grids, there is also a disparity in market mechanisms that complicates attempts 
to bridge the gap in coordination between these sectors [19].

Interest in developing auction-based pricing mechanisms for pipeline capacity that are similar to what is used in wholesale electricity markets goes back nearly 30 years, as evidenced by the 1987 FERC report [20]. In that report, a linear programming model for auctioning pipeline transportation rights was proposed, with primary auctions to be conducted as often as daily. More frequent secondary auctions for re-selling of capacity rights were envisioned as well. Many of the ideas in the 1987 proposal remain relevant and deserve to be re-examined in light of noted trends in the natural gas industry, improved optimization techniques, and the significant experience gained through successful implementation of auction-based market mechanisms over the past two decades in the power industry worldwide.

In this paper, we begin a study of the properties of locational marginal prices of natural gas (gLMPs) based on the single price auction mechanism and the physics of natural gas flows in pipeline networks. In contrast to previous studies [14], [21], we do not linearize gas flow equations and thus retain the impact of non-linearities on price formation. Our use of nonlinear models is justified by recent advances in computing technology and optimization methods [22], [18]. The results presented in this paper are limited to formulations for steady-state flow, and show promise for extension to dynamic price models that capture the effect of flow transients and line pack. We adopt an engineering approach developed for large-scale control system modeling of gas pipelines [23], [24], so that constraints on flow and energy usage by compressors can be described. An optimization formulation that maximizes social welfare is presented in which variable transportation costs are factored into the pricing mechanism. While marginal pricing and economic spot markets for gas have been studied [25], the gLMPs derived here provide price signals that reflect the physical ability to transport gas through a pipeline system. We present an engineering economic analysis of price differentials created through the proposed market mechanism, and prove revenue adequacy for the market administrator.

The rest of the manuscript is structured as follows. In Section II, we describe the engineering model that we use to represent a gas pipeline network under steady-state flow conditions. Section III follows with the derivation of the maximum social welfare optimization problem, and a derivation of the optimality conditions. In Section IV, we derive expressions for locational marginal gas prices, and prove revenue adequacy in Section V. We then present and interpret two computational examples in Section VI, and conclude with a discussion of promising directions in VII.

\section{Engineering Model of a Gas Pipeline Network Under STEAdY-STATE Flow CONDitions}

We represent a gas pipeline network as a metric graph $\Gamma=(\mathcal{V}, \mathcal{E}, B)$ where $\mathcal{V}$ is the set of vertices (or nodes) and $\mathcal{E} \subset V \times V$ is the set of directed edges $(i, j) \in \mathcal{E}$ that connect the nodes $i, j \in \mathcal{V}$. Every edge $(i, j) \in \mathcal{E}$ is associated with a resistance term $\beta_{i j}=B(i, j)$ given by the map $B: \mathcal{E} \rightarrow \mathbb{R}_{+}$, where $\mathbb{R}_{+}=\{x \in \mathbb{R}: x \geq 0\}$. This map defines the graph edge metric that quantifies physical resistance to flow through each pipe. We let $V=|\mathcal{V}|$ and $E=|\mathcal{E}|$ denote the number of nodes and of edges, respectively. In addition to network nodes, we introduce the set $\mathcal{G}$ of gNodes, which enumerate the notional receipt or delivery points associated with network nodes in $\mathcal{V}$. Each supplier is considered to be injecting gas at a unique gNode, and each off-taker withdraws gas at a unique gNode. Each gNode $k \in \mathcal{G}$ can represent only one supplier or off-taker, and is associated with a unique network node $j(k) \in \mathcal{V}$. The incoming and outgoing neighborhoods of a node $j \in \mathcal{V}$, and the set of gNodes connected to $j \in \mathcal{V}$, are respectively denoted by

$$
\begin{aligned}
& \partial_{+} j=\{i \in \mathcal{V} \mid(i, j) \in \mathcal{E}\} \subset \mathcal{V}, \\
& \partial_{-} j=\{k \in \mathcal{V} \mid(j, k) \in \mathcal{E}\} \subset \mathcal{V}, \\
& \partial_{g} j=\{k \in \mathcal{G} \mid j(k) \in \mathcal{V}\} \subset \mathcal{G} .
\end{aligned}
$$

In steady flow, the system can be characterized by pressures $p_{j}$ at each node $j \in \mathcal{V}$, or by flow $\phi_{i j}$ on each edge $(i, j) \in \mathcal{E}$ and pressure at one node (e.g. a "slack" node) $i \in \mathcal{V}$. In addition, every node in $\mathcal{V}$ is associated with a boundary condition, which is either a fixed pressure (if it is a slack node), or the total mass flow injection $q_{j}$ (for all other non-slack nodes). This mass injection is equal to the supply minus the consumption at that node, as expressed by

$$
q_{j}=\sum_{k \in \partial_{g} j}\left(s_{k}-d_{k}\right)
$$

The balance of incoming and outgoing physical mass flows and injection of gas into the network must be preserved for each network node $j \in \mathcal{V}$. This is expressed as a constraint for all non-slack nodes by

$$
\sum_{i \in \partial_{+} j} \bar{\phi}_{i j}-\sum_{k \in \partial_{-} j} \underline{\phi}_{j k}=-\sum_{k \in \partial_{g} j}\left(s_{k}-d_{k}\right), \quad \forall j \in \mathcal{V} .
$$

The pressure of gas flowing through the system is boosted by compressor stations, or compressors for short. In our model, these compressors are located at the interfaces between nodes and adjoining pipes. Each is able to boost flow going from a node into an adjoining pipe. We define two sets of these compressors, $\underline{\mathcal{C}} \subset \mathcal{E}$ and $\bar{C} \subset \mathcal{E}$, where $(\underline{i, j}) \in \underline{\mathcal{C}}$ denotes a compressor located near node $i \in \mathcal{V}$ that boosts flow into edge $(i, j) \in \mathcal{E}$ in the positive direction, while $(\overline{i, j}) \in \bar{C}$ denotes a compressor located near node $j \in \mathcal{V}$ that boosts flow into edge $(i, j) \in \mathcal{E}$ in the negative direction. Compression is then modeled as a multiplicative ratio $\underline{\alpha}_{i j} \in \mathbb{R}_{+}$for $(i, j) \in \underline{\mathcal{C}}$ and $\bar{\alpha}_{i j} \in \mathbb{R}_{+}$for $(\overline{i, j}) \in \overline{\mathcal{C}}$.

Each compression ratio provides a relation between nodal pressure at compressor suction and pipe boundary pressure at compressor discharge. Thus, the suction pressure $p_{j}$ of a compressor $(\underline{j, k})$ for $k \in \partial_{-} j$ is related to the pipe boundary 
pressure $\underline{p}_{j k}$ at the $j$ end of pipe $(j, k)$ by

$$
\underline{p}_{j k}=\underline{\alpha}_{j k} p_{j}, \quad \forall(j, k) \in \mathcal{E} .
$$

Similarly, the suction pressure of a compressor $(\overline{i, j})$ for $i \in$ $\partial_{+} j$ is related to the pipe boundary pressure $\bar{p}_{i j}$ at the $j$ end of pipe $(i, j)$ by

$$
\bar{p}_{i j}=\bar{\alpha}_{i j} p_{j}, \quad \forall(i, j) \in \mathcal{E} .
$$

These pressure variables are illustrated in Figure 1. We assume that compressor stations are designed and operated only to boost pressure, so that $\underline{\alpha}_{i j}, \bar{\alpha}_{i j} \geq 1$.

Without loss of generality, we can assume that each compressor can be powered by burning a portion of gas flowing through it, or by using an equivalent amount of electric energy in a known proportion that is specific for each compressor. The energy consumption rates of compressors powered by gas are then approximated by

$$
\begin{array}{ll}
\underline{E}_{i j}=\underline{\eta}_{i j}\left|\underline{\phi}_{i j}\right|\left(\left(\underline{\alpha}_{i j}^{2 m}\right)-1\right), & (\underline{i, j}) \in \underline{\mathcal{C}}, \\
\bar{E}_{i j}=\bar{\eta}_{i j}\left|\bar{\phi}_{i j}\right|\left(\left(\bar{\alpha}_{i j}^{2 m}\right)-1\right), & (\overline{i, j}) \in \overline{\mathcal{C}},
\end{array}
$$

with $0<m=\left(\gamma_{g}-1\right) / \gamma_{g}<1$ where $\gamma_{g}$ is the heat capacity ratio of the gas [17]. With the appropriate constants $\underline{\eta}_{i j}$ and $\bar{\eta}_{i j}$, the energy consumption rates (8)-(9) can be expressed in the same units of mass flow rate as $\phi_{i j}$ to reflect the gas consumed as fuel for compression.

The differences between discharge flow and suction flow of compressors powered by gas are given by (8)-(9). For each compressor, we introduce the parameter $\varepsilon \in\{0,1\}$, where $\varepsilon=1$ when the compressor is powered by gas and $\varepsilon=0$ indicates that the compressor is powered by electricity. Under this assumption, the suction flow $\underline{\phi}_{j k}$ of a compressor $(j, k)$ for $k \in \partial_{-} j$ (flow entering the compressor $(j, k)$ from node $j$ ) is related to the discharge flow $\phi_{j k}$ (which is uniform along pipe $(j, k))$ according to

$$
\phi_{j k}=\underline{\phi}_{j k}-\underline{\varepsilon}_{j k} \underline{E}_{j k}, \quad \forall(j, k) \in \mathcal{E} .
$$

Similarly, the suction flow $\bar{\phi}_{i j}$ of a compressor $(\overline{i, j})$ for $i \in$ $\partial_{+} j$ (flow entering the compressor $(\overline{i, j})$ from node $j$ ) is related to the discharge flow $\phi_{i j}$ (which is uniform along pipe $(i, j))$ according to

$$
\phi_{i j}=\bar{\phi}_{i j}+\bar{\varepsilon}_{j k} \bar{E}_{i j}, \quad \forall(i, j) \in \mathcal{E} .
$$

These flow variables are illustrated in Figure 1.

We model the relationship between flow and boundary pressures in a pipe $(i, j)$ by assuming isothermal, steadystate flow in a horizontal pipe with higher order inertial terms omitted. Dissipative effects are aggregated using the DarcyWiesbach law with resistance parameter $\beta_{i j}$, which depends on pipe length $L_{i j}$, diameter $D_{i j}$, cross-sectional area $A_{i j}$, Colebrook-White friction factor $f_{i j}$, and the wave speed $a$. We assume a constant gas compressibility and temperature, so that this speed of sound $a=\sqrt{Z R T}$ is constant throughout
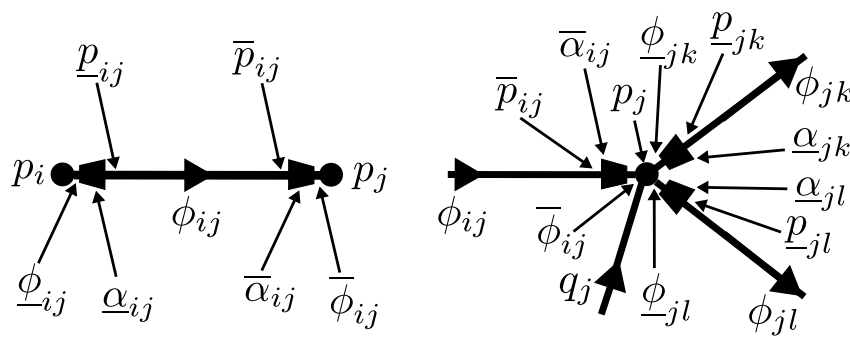

Figure 1: Nodal pressures $p_{j}$, edge flows $\phi_{i j}$, edge endpoint variables $\underline{p}_{i j}, \underline{\phi}_{i j}, \bar{p}_{i j}, \bar{\phi}_{i j}$, and actuators $\underline{\alpha}_{i j}, \bar{\alpha}_{i j}$ for an edge (left) and for a joint (right).

the system. This yields

$$
\underline{p}_{i j}^{2}-\bar{p}_{i j}^{2}=\beta_{i j} \phi_{i j}\left|\phi_{i j}\right|, \beta_{i j}=a^{2} \frac{f_{i j} L_{i j}}{D_{i j} A_{i j}^{2}}, \forall(i, j) \in \mathcal{E} .
$$

\section{MaXimum Social Welfare Optimization}

We formulate the economic optimization problem of maximizing the sum of the consumer and producer surplus otherwise known as social welfare, with an objective function similar to previous studies [20], [21]. In this formulation, the compressor stations are assumed to consume a portion of the gas flowing through the system to obtain power. Thus, the operating cost to the pipeline system operator is minimized as well.

The objective function is formulated as follows. All suppliers and offtakers are characterized by offer prices $c_{k}^{s}$ and bid prices $c_{k}^{o}$, respectively, specified for each gNode $k \in \mathcal{G}$. Some gNodes are assumed to account for demand interruptions that are represented by supplies offered at sufficiently high price. Including such supplies helps to assure the feasibility of the optimization problem. We denote the price of electricity delivered to the gas network node $i \in \mathcal{V}$ by $\lambda_{i}^{e}$, so the price components $\underline{C}_{i j}^{e}$ and $\bar{C}_{i j}^{e}$ of electric compression at either end of a pipe $(i, j) \in \mathcal{E}$ can be expressed as

$$
\underline{C}_{i j}^{e}=\lambda_{i}^{e}\left(1-\underline{\varepsilon}_{i j}\right) \underline{E}_{i j}, \quad \bar{C}_{i j}^{e}=\lambda_{j}^{e}\left(1-\bar{\varepsilon}_{i j}\right) \bar{E}_{i j} .
$$

The maximum social welfare objective function is therefore

$$
\begin{aligned}
J_{\mathrm{MSW}} & \triangleq \sum_{k \in \mathcal{G}} c_{k}^{o} d_{k}-\sum_{k \in \mathcal{G}} c_{k}^{s} s_{k} \\
& -\sum_{(i, j) \in \mathcal{E}}\left(\lambda_{i}^{e}\left(1-\underline{\varepsilon}_{i j}\right) \underline{E}_{i j}+\lambda_{j}^{e}\left(1-\bar{\varepsilon}_{i j}\right) \bar{E}_{i j}\right)
\end{aligned}
$$

In addition to the equality constraints defined by equations (5)-(12), engineering considerations require several inequality constraints. Appropriate pressure limits must be adhered to for two reasons. Upper bounds are associated with engineering limitations called the maximum allowable operating pressure (MAOP), which depend on each pipe,

$$
\underline{p}_{i j} \leq p_{i j}^{\max }, \quad \bar{p}_{i j} \leq p_{i j}^{\max }, \quad \forall(i, j) \in \mathcal{E} .
$$

Lower bounds are associated with contractual obligations to 
$\max \quad$ Social Welfare: $J_{M S W} \triangleq \sum_{k \in \mathcal{G}} c_{k}^{o} d_{k}-\sum_{k \in \mathcal{G}} c_{k}^{s} s_{k}$

$$
-\sum_{j \in \mathcal{V}} \sum_{i \in \partial_{+} j} \lambda_{j}^{e}\left(1-\bar{\varepsilon}_{i j}\right) \bar{\eta}_{i j}\left|\phi_{i j}\right|\left(\left(\bar{\alpha}_{i j}^{2 m}\right)-1\right)-\sum_{j \in \mathcal{V}} \sum_{k \in \partial_{-}} \lambda_{j}^{e}\left(1-\underline{\varepsilon}_{j k}\right) \underline{\eta}_{j k}\left|\phi_{j k}\right|\left(\left(\underline{\alpha}_{j k}^{2 m}\right)-1\right)
$$

s.t. $\quad$ Flow balance: $\quad \sum_{k \in \partial_{-} j} \phi_{j k}-\sum_{i \in \partial_{+} j} \phi_{i j}=\sum_{k \in \partial_{g} j}\left(s_{k}-d_{k}\right)$

$$
-\sum_{i \in \partial_{+} j} \bar{\varepsilon}_{i j} \bar{\eta}_{i j}\left|\phi_{i j}\right|\left(\left(\bar{\alpha}_{i j}^{2 m}\right)-1\right)-\sum_{k \in \partial_{-}} \underline{\varepsilon}_{j k} \underline{\eta}_{j k}\left|\phi_{j k}\right|\left(\left(\underline{\alpha}_{j k}^{2 m}\right)-1\right), \quad \forall j \in \mathcal{V}, \quad \lambda_{j}
$$

Pressure balance: $\quad\left(\underline{\alpha}_{i j} p_{i}\right)^{2}-\left(\bar{\alpha}_{i j} p_{j}\right)^{2}=\beta_{i j} \phi_{i j}\left|\phi_{i j}\right|$,

$\forall(i, j) \in \mathcal{E}, \quad \mu_{i j}$

Pressure limits: $\underline{\alpha}_{i j} p_{i} \leq p_{i j}^{\max }, \quad \bar{\alpha}_{i j} p_{j} \leq p_{i j}^{\max }$,

$\forall(i, j) \in \mathcal{E}, \quad \underline{\xi}_{i j}^{\max }, \bar{\xi}_{i j}^{\max }$

$$
p_{j}^{\min } \leq p_{j}
$$

Boost upper limits: $\underline{\eta}_{i j}\left|\phi_{i j}\right|\left(\left(\underline{\alpha}_{i j}^{2 m}\right)-1\right) \leq \underline{E}_{i j}^{\max }, \quad \bar{\eta}_{i j}\left|\phi_{i j}\right|\left(\left(\bar{\alpha}_{i j}^{2 m}\right)-1\right) \leq \bar{E}_{i j}^{\max }$

$$
\forall j \in \mathcal{V}, \quad \xi_{j}^{\min }
$$

Boost lower limits: $\underline{\alpha}_{i j}, \bar{\alpha}_{i j} \geq 1$

$$
\forall(i, j) \in \mathcal{E}, \quad \underline{\gamma}_{i j}, \bar{\gamma}_{i j}
$$

Supply limits: $s_{k}^{\min } \leq s_{k} \leq s_{k}^{\max }$

Demand limits: $d_{k}^{\min } \leq d_{k} \leq d_{k}^{\max }$

$\forall(i, j) \in \mathcal{E}, \quad \underline{\theta}_{i j}, \bar{\theta}_{i j}$

$\forall k \in \mathcal{G}, \quad \sigma_{k}^{\min }, \sigma_{k}^{\max }$

$\forall k \in \mathcal{G}, \quad \zeta_{k}^{\min }, \zeta_{k}^{\max }$

$$
\begin{aligned}
L= & \sum_{k \in \mathcal{G}}\left(c_{k}^{s} s_{k}-c_{k}^{o} d_{k}\right)+\sum_{j \in \mathcal{V}} \sum_{i \in \partial_{+} j} \lambda_{j}^{e}\left(1-\bar{\varepsilon}_{i j}\right) \bar{\eta}_{i j}\left|\phi_{i j}\right|\left(\left(\bar{\alpha}_{i j}^{2 m}\right)-1\right)+\sum_{j \in \mathcal{V}} \sum_{k \in \partial_{-j}} \lambda_{j}^{e}\left(1-\underline{\varepsilon}_{j k}\right) \underline{\eta}_{j k}\left|\phi_{j k}\right|\left(\left(\underline{\alpha}_{j k}^{2 m}\right)-1\right) \\
& +\sum_{(i, j) \in \mathcal{E}} \mu_{i j}\left(\left(\underline{\alpha}_{i j} p_{i}\right)^{2}-\left(\bar{\alpha}_{i j} p_{j}\right)^{2}-\beta_{i j} \phi_{i j}\left|\phi_{i j}\right|\right)+\sum_{j \in \mathcal{V}} \lambda_{j}\left(\sum_{k \in \partial_{-j}} \phi_{j k}-\sum_{i \in \partial_{+} j} \phi_{i j}-\sum_{k \in \partial_{g} j}\left(s_{k}-d_{k}\right)\right) \\
& +\sum_{j \in \mathcal{V}} \lambda_{j}\left(\sum_{i \in \partial_{+} j} \bar{\varepsilon}_{i j} \bar{\eta}_{i j}\left|\phi_{i j}\right|\left(\left(\bar{\alpha}_{i j}^{2 m}\right)-1\right)+\sum_{k \in \partial_{-}} \underline{\varepsilon}_{j k} \underline{\eta}_{j k}\left|\phi_{j k}\right|\left(\left(\underline{\alpha}_{j k}^{2 m}\right)-1\right)\right)+\sum_{j \in \mathcal{V}} \xi_{j}^{\min }\left(p_{j}^{\min }-p_{j}\right) \\
& +\sum_{(i, j) \in \mathcal{E}}\left(\underline{\xi}_{i j}^{\max }\left(\underline{\alpha}_{i j} p_{i}-p_{i j}^{\max }\right)+\bar{\xi}_{i j}^{\max }\left(\bar{\alpha}_{i j} p_{j}-p_{i j}^{\max }\right)\right)+\sum_{(i, j) \in \mathcal{E}}\left(\underline{\theta}_{i j}\left(1-\underline{\alpha}_{i j}\right)+\bar{\theta}_{i j}\left(1-\bar{\alpha}_{i j}\right)\right) \\
& +\sum_{(i, j) \in \mathcal{E}}\left(\underline{\gamma}_{i j}\left(\underline{\eta}_{i j}\left|\phi_{i j}\right|\left(\left(\underline{\alpha}_{i j}^{2 m}\right)-1\right)-\underline{E}_{i j}^{\max }\right)+\bar{\gamma}_{i j}\left(\bar{\eta}_{i j}\left|\phi_{i j}\right|\left(\left(\bar{\alpha}_{i j}^{2 m}\right)-1\right)-\bar{E}_{i j}^{\max }\right)\right) \\
& +\sum_{k \in \mathcal{G}}\left(\sigma_{k}^{\min }\left(s_{k}^{\min }-s_{k}\right)+\sigma_{k}^{\max }\left(s_{k}-s_{k}^{\max }\right)+\zeta_{k}^{\min }\left(d_{k}^{\min }-d_{k}\right)+\zeta_{k}^{\max }\left(d_{k}-d_{k}^{\max }\right)\right)
\end{aligned}
$$

maintain minimum pressures so that customers at each node can withdraw gas from the line, and are given by

$$
p_{j} \geq p_{j}^{\min }, \quad \forall j \in \mathcal{V} .
$$

Another key engineering limitation is the maximum power of compressors used to actuate flow through the system. Therefore, upper limits on $\underline{E}_{i j}$ and $\bar{E}_{i j}$ are given by

$$
\underline{E}_{i j} \leq \underline{E}_{i j}^{\max }, \quad \bar{E}_{i j} \leq \bar{E}_{i j}^{\max }, \quad \forall(i, j) \in \mathcal{E} .
$$

We have assumed that compressor stations are designed and operated only to boost pressure, so that

$$
\underline{\alpha}_{i j} \geq 1, \quad \bar{\alpha}_{i j} \geq 1, \quad \forall(i, j) \in \mathcal{E} .
$$

Supplier limitations and offtaker capacities at each gNode are subject to minimum and maximum constraints

$$
s_{k}^{\min } \leq s_{k} \leq s_{k}^{\max }, \quad d_{k}^{\min } \leq d_{k} \leq d_{k}^{\max }, \quad \forall k \in \mathcal{G} .
$$

With the above collection of engineering and physical constraints, the maximum social welfare problem is

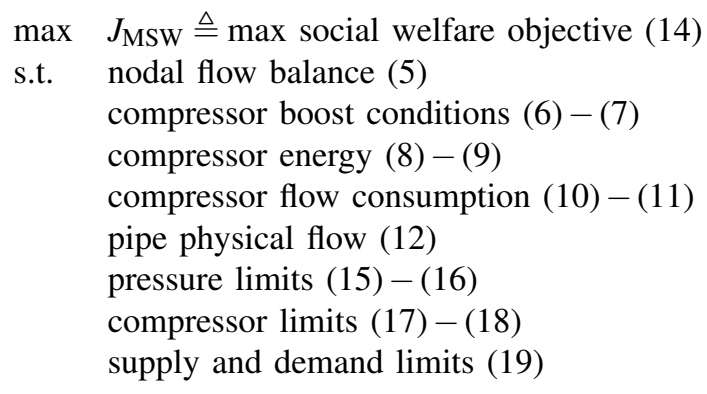

We make several substitutions in problem (20) to create a new, simpler problem (21). We substitute for $\underline{\phi}_{i j}$ and $\bar{\phi}_{i j}$ using equations (10)-(11), and for $\underline{p}_{i j}$ and $\bar{p}_{i j}$ using equations (6)-(7). We may approximate $\phi_{i j}$ and $\bar{\phi}_{i j}$ by $\phi_{i j}$ in constraints 


$$
\begin{aligned}
& s_{k}: \quad 0=c_{k}^{s}-\lambda_{j(k)}+\sigma_{k}^{\max }-\sigma_{k}^{\min } \\
& d_{k}: \quad 0=-c_{k}^{o}+\lambda_{j(k)}+\zeta_{k}^{\max }-\zeta_{k}^{\min } \\
& p_{j}: \quad 0=\sum_{i \in \partial_{+} j}\left(-2 \mu_{i j} \bar{\alpha}_{i j}^{2} p_{j}+\bar{\xi}_{i j}^{\max } \bar{\alpha}_{i j}\right)+\sum_{k \in \partial_{-j}}\left(2 \mu_{j k} \underline{\alpha}_{j k}^{2} p_{j}+\underline{\xi}_{j k}^{\max } \underline{\alpha}_{j k}\right)-\xi_{j}^{\min } \\
& \phi_{i j}: \quad 0=\left(\lambda_{i}-\lambda_{j}\right)-2 \beta_{i j} \mu_{i j}\left|\phi_{i j}\right|+\left(\bar{\gamma}_{i j}+\bar{\varepsilon}_{i j} \lambda_{j}+\lambda_{j}^{e}\left(1-\bar{\varepsilon}_{i j}\right)\right) \bar{\eta}_{i j}\left(\left(\bar{\alpha}_{i j}^{2 m}\right)-1\right) \operatorname{sign}\left(\phi_{i j}\right) \\
& +\left(\underline{\gamma}_{i j}+\underline{\varepsilon}_{i j} \lambda_{i}+\lambda_{i}^{e}\left(1-\underline{\varepsilon}_{i j}\right)\right) \underline{\eta}_{i j}\left(\left(\underline{\alpha}_{i j}^{2 m}\right)-1\right) \operatorname{sign}\left(\phi_{i j}\right) \\
& \underline{\alpha}_{i j}: \quad 0=2 \underline{\alpha}_{i j} \mu_{i j} p_{i}^{2}+2 m\left(\underline{\gamma}_{i j}+\underline{\varepsilon}_{i j} \lambda_{i}+\left(1-\underline{\varepsilon}_{i j}\right) \lambda_{i}^{e}\right) \underline{\eta}_{i j}\left|\phi_{i j}\right| \underline{\alpha}_{i j}^{2 m-1}+p_{i} \underline{\xi}_{i j}^{\max }-\underline{\theta}_{i j} \\
& \forall(i, j) \in \mathcal{E} \\
& \bar{\alpha}_{i j}: \quad 0=-2 \bar{\alpha}_{i j} \mu_{i j} p_{j}^{2}+2 m\left(\bar{\gamma}_{i j}+\bar{\varepsilon}_{i j} \lambda_{j}+\lambda_{j}^{e}\left(1-\bar{\varepsilon}_{i j}\right)\right) \bar{\eta}_{i j}\left|\phi_{i j}\right| \bar{\alpha}_{i j}^{2 m-1}+p_{j} \bar{\xi}_{i j}^{\max }-\bar{\theta}_{i j}=0 \\
& \forall(i, j) \in \mathcal{E} \\
& \forall(i, j) \in \mathcal{E}
\end{aligned}
$$

(8)-(9), because compressor flow consumptions $\underline{E}_{i j}$ and $\bar{E}_{i j}$ are small compared to flow $\phi_{i j}$. We then substitute for $\underline{E}_{i j}$ and $\bar{E}_{i j}$ using these modified constraints. This yields the formulation given as problem (21), where the appropriate Lagrange multiplier is denoted to the right of each constraint.

In the problem (21), only nodal pressures $p_{j}$ for $j \in \mathcal{V}$ and edge flows $\phi_{i j}$ for $(i, j) \in \mathcal{E}$ appear, instead of the boundary variables that are used in the formulation (20). Note that in the solution, only one of the compressors attached at the boundaries of a pipe can be working. Although this can be shown using an economic argument, it is an engineering requirement, because gas cannot flow from discharge to suction through a working compressor. The Lagrangian for the problem is given in equation (22). Because our objective is a maximization, we reverse the sign of the objective function in the Lagrangian. The Karush-Kuhn Tucker (KKT) optimality conditions are obtained by taking partial derivatives of $L$ with respect to the optimization variables and setting to zero [26]. These KKT conditions are given in equations (23) for the variables (denoted at left). Complementary slackness also requires the conditions

$$
\begin{array}{lr}
\underline{\xi}_{i j}^{\max }\left(\underline{\alpha}_{i j} p_{i}-p_{i j}^{\max }\right)=0, & \forall(i, j) \in \mathcal{E} \\
\bar{\xi}_{i j}^{\max }\left(\bar{\alpha}_{i j} p_{j}-p_{i j}^{\max }\right)=0, & \forall(i, j) \in \mathcal{E} \\
\xi_{j}^{\min }\left(p_{j}^{\min }-p_{j}\right)=0, & \forall j \in \mathcal{V} \\
\underline{\gamma}_{i j}\left(\underline{\eta}_{i j}\left|\phi_{i j}\right|\left(\left(\underline{\alpha}_{i j}^{2 m}\right)-1\right)-\underline{E}_{i j}^{\max }\right)=0, & \forall(i, j) \in \mathcal{E} \\
\bar{\gamma}_{i j}\left(\bar{\eta}_{i j}\left|\phi_{i j}\right|\left(\left(\bar{\alpha}_{i j}^{2 m}\right)-1\right)-\bar{E}_{i j}^{\max }\right)=0, & \forall(i, j) \in \mathcal{E} \\
\underline{\theta}_{i j}\left(1-\underline{\alpha}_{i j}\right)=0, \quad \bar{\theta}_{i j}\left(1-\bar{\alpha}_{i j}\right)=0, & \forall(i, j) \in \mathcal{E} \\
\sigma_{k}^{\min }\left(s_{k}^{\min }-s_{k}\right)=0, & \forall k \in \mathcal{G} \\
\sigma_{k}^{\max }\left(s_{k}-s_{k}^{\max }\right)=0, & \forall k \in \mathcal{G} \\
\zeta_{k}^{\min }\left(d_{k}^{\min }-d_{k}\right)=0, & \forall k \in \mathcal{G} \\
\zeta_{k}^{\max }\left(d_{k}-d_{k}^{\max }\right)=0 . & \forall k \in \mathcal{G}
\end{array}
$$

Furthermore, note that inequality constraints have been written in the form $g(x) \leq 0$, so that dual feasibility requires that the multipliers on these constraints are positive, i.e.,

$$
\begin{array}{r}
\xi_{j}^{\min }, \underline{\xi}_{i j}^{\max }, \bar{\xi}_{i j}^{\max }, \zeta_{i j}^{\min }, \zeta_{i j}^{\max }, \sigma_{i j}^{\min }, \sigma_{i j}^{\max } \geq 0, \\
\underline{\gamma}_{i j}, \bar{\gamma}_{i j}, \underline{\theta}_{i j}, \bar{\theta}_{i j} \geq 0 .
\end{array}
$$

\section{Analysis of Locational Prices}

By definition, shadow prices for the nodal balance constraint reflect the change in the objective function (social welfare) in response to the infinitesimal increase in natural gas consumption at location $j$, and can therefore be interpreted as locational marginal prices of natural gas (or gLMPs). It is important to note that the necessary KKT conditions on which we rely in this analysis do not by themselves guarantee that shadow prices will be equal to the Lagrange multipliers $\lambda_{j}$ specified in KKT conditions. A proof that shadow prices are equivalent to Lagrange multipliers makes the latter economically meaningful, and is required to justify an interpretation of the Lagrange multipliers $\lambda_{j}$ as gLMPs.

Such a proof usually follows a two-step process: 1) establishing the equivalence between finding an optimum solution in a constraint optimization problem and finding a saddle-point of that problem's Lagrangian; and 2) proving differentiability (or more precisely absolute continuity) of the value of the Lagrangian as a function of the constraint limit. The second part of the proof relies on the envelope theorem [27]. It is important to note that this two-step process does not require a convexity assumption. In fact, differentiability of the objective function and functions that define constraints in the neighborhood of the optimum are sufficient to claim that the optimal value of the objective function is an absolutely continuous function of the constraint limit, and to equate the shadow price to a Lagrange multiplier. Indeed, the envelope theorem for saddle-point problems (Theorem 4 in [27]) requires no convexity and holds under the assumption that the Lagrangian is an absolutely continuous function of primal and dual variables. This holds in the present case. Furthermore, demonstrating equivalence between a constraint optimization problem and a saddle-point problem for the Lagrangian does not require a convexity assumption. For example, differentiability of the optimization problem in the neighborhood of the optimum is sufficient to demonstrate such equivalence (see Saddle Point Theorem on p. 76 of [28]). So, although the optimization problem above is non-convex, it is justifiable to interpret the values of $\lambda_{j}$ as gLMPs.

In the following, we proceed to analyze the Lagrange 
multipliers to explore mathematical relationships between gLMPs and other characteristics of the system, and to provide economic interpretation of these relationships. First, we establish the relationship between gLMPs, supply offers, and offtaker bids. These basic relationships that follow directly from (23a)-(23b) can be expressed as

$$
\begin{aligned}
& \lambda_{j(k)}=c_{k}^{s}+\sigma_{k}^{\max }-\sigma_{k}^{\min }, \\
& \lambda_{j(k)}=c_{k}^{o}-\zeta_{k}^{\max }+\zeta_{k}^{\min } .
\end{aligned}
$$

We can then classify suppliers and offtakers according to the relation between their offers/bids and the nodal gLMP.

$$
\begin{array}{cl}
\text { Marginal Supplier: } & \lambda_{j(k)}=c_{k}^{s}, \\
\sigma_{k}^{\max }=\sigma_{k}^{\min }=0, & s_{k}^{\min }<s_{k}<s_{k}^{\max } \\
\text { Supra-Marginal Supplier: } & \lambda_{j(k)}<c_{k}^{s}, \\
\sigma_{k}^{\max }=0, \sigma_{k}^{\min }=c_{k}^{s}-\lambda_{j(k)}, & s_{k}=s_{k}^{\min } \\
\text { Infra-Marginal Supplier: } & \lambda_{j(k)}>c_{k}^{s}, \\
\sigma_{k}^{\min }=0, \sigma_{k}^{\max }=\lambda_{j(k)}-c_{k}^{s}, & s_{k}=s_{k}^{\max } . \\
\text { Marginal Offtaker: } & \lambda_{j(k)}=c_{k}^{o}, \\
\zeta_{k}^{\max }=\zeta_{k}^{\min }=0, & d_{k}^{\min }<d_{k}<d_{k}^{\max } \\
\text { Supra-Marginal Offtaker: } & \lambda_{j(k)}<c_{k}^{o}, \\
\zeta_{k}^{\min }=0, \zeta_{k}^{\max }=c_{k}^{o}-\lambda_{j(k)}, & d_{k}=d_{k}^{\max } . \\
\text { Infra-Marginal Offtaker: } & \lambda_{j(k)}>c_{k}^{o}, \\
\zeta_{k}^{\max }=0, \zeta_{k}^{\min }=\lambda_{j(k)}-c_{k}^{o}, d_{k}=d_{k}^{\min } .
\end{array}
$$

Offers of infra-marginal suppliers and bids of supra-marginal offtakers are taken at maximum level because infra-marginal supply is offered below cleared price and a supra-marginal offtaker is willing to buy above the cleared prices. In contrast, offers of supra-marginal suppliers and infra-marginal off-takes are taken at the minimum level, because such supplies are offered above cleared prices and offtakers' willingness to pay is below clearing price.

Next we explore equations (23d) - (23f). Here we make an assumption (which could be confirmed by a formal proof) that in an optimal solution of problem (21), the compression in any given pipe is only applied at the sending end and never at the receiving end, where sending and receiving are defined according to the actual direction of the flow. Thus, if $\phi_{i j}>0$, then $\bar{\alpha}_{i j}=1$ and if $\phi_{i j}<0$, then $\underline{\alpha}_{i j}=1$. With this assumption we restate (23d) - (23f) for the case $\phi_{i j}>0$.

$$
\begin{aligned}
& \lambda_{j}-\lambda_{i}=\left(\underline{\gamma}_{i j}+\underline{\varepsilon}_{i j} \lambda_{i}+\lambda_{i}^{e}\left(1-\underline{\varepsilon}_{i j}\right)\right) \underline{\eta}_{i j}\left(\left(\underline{\alpha}_{i j}^{2 m}\right)-1\right) \\
&-2 \beta_{i j} \mu_{i j} \phi_{i j} \\
& 2 \underline{\alpha}_{i j} \mu_{i j} p_{i}^{2}=-2 m\left(\underline{\gamma}_{i j}+\underline{\varepsilon}_{i j} \lambda_{i}+\left(1-\underline{\varepsilon}_{i j}\right) \lambda_{i}^{e}\right) \underline{\eta}_{i j} \phi_{i j} \underline{\alpha}_{i j}^{2 m-1} \\
&-p_{i} \underline{\xi}_{i j}^{\max }+\underline{\theta}_{i j} \\
& 2 \mu_{i j} p_{j}^{2}=2 m\left(\bar{\varepsilon}_{i j} \lambda_{j}+\lambda_{j}^{e}\left(1-\bar{\varepsilon}_{i j}\right)\right) \bar{\eta}_{i j} \phi_{i j}+p_{j} \bar{\xi}_{i j}^{\max }-\bar{\theta}_{i j}
\end{aligned}
$$

Solving equation (31) for $\mu_{i j}$ and substituting this into
(30) yields an expression for the basis differential (price difference) between two neighboring nodes given by

$$
\lambda_{j}-\lambda_{i}=\underline{\delta}_{i j}+\underline{\Delta}_{i j}^{c}+\underline{\Delta}_{i j}^{p}
$$

where $\underline{\delta}_{i j}$ is the compression component of the basis differential, which is equal to the product of the purchase price of energy and marginal energy consumed by the compressor to deliver an incremental unit of gas along the pipe $(i, j)$. In addition, the basis differential may include two congestion components, denoted by $\underline{\Delta}_{i j}^{c}$ and $\underline{\Delta}_{i j}^{p}$, which are associated with a compressor constrained at its maximum capacity and discharge pressure constrained at the MAOP of the pipe, respectively. These components are given by

$$
\begin{aligned}
\underline{\delta}_{i j} & =\left(\underline{\varepsilon}_{i j} \lambda_{i}+\lambda_{i}^{e}\left(1-\underline{\varepsilon}_{i j}\right)\right) \underline{M}_{i j}-\underline{\theta}_{i j} \frac{\beta_{i j} \phi_{i j}}{\underline{\alpha}_{i j} p_{i}^{2}}, \\
\underline{\Delta}_{i j}^{c} & =\underline{\gamma}_{i j} \underline{M}_{i j}, \\
\underline{\Delta}_{i j}^{p} & =\underline{\xi}_{i j}^{\max } \frac{\beta_{i j} \phi_{i j}}{\underline{\alpha}_{i j} p_{i}}, \\
\underline{M}_{i j} & =\underline{\eta}_{i j}\left(\underline{\alpha}_{i j}^{2 m}-1\right)+2 m \underline{\eta}_{i j} \underline{\alpha}_{i j}^{2 m} \frac{\beta_{i j} \phi_{i j}^{2}}{\left(\underline{\alpha}_{i j} p_{i}\right)^{2}},
\end{aligned}
$$

where $\underline{M}_{i j}$ is the energy used by compressor to move marginal volume of gas along the pipe. If the compressor operates, then $\underline{\alpha}_{i j}>1$ and $\underline{\theta}_{i j}=0$, hence the compression component is strictly positive. Furthermore, in this case the relationship between the flow and compression given by the pressure balance equation (21c) is

$$
\underline{\alpha}_{i j}^{2}=\frac{\beta_{i j} \phi_{i j}^{2}+p_{j}^{2}}{p_{i}^{2}} .
$$

It is straightforward to demonstrate that the compression component $\underline{\delta}_{i j}$ is monotonically increasing with the gas flow.

Two congestion components in equation (33) correspond to two possible conditions that may lead to congestion. Specifically, congestion occurs when the compressor operates at its capacity limit, resulting in $\underline{\gamma}_{i j}>0$, or when the pressure reaches its upper limit, resulting in $\underline{\xi}_{i j}^{\max }>0$. In all cases, the congestion component is non-negative. Thus, if the compression is applied, the basis differential in equation (33) is strictly positive, which indicates that the gas would flow from the lower price node to the higher price node. In the absence of compression, the compression component gains a negative term of the form

$$
-\underline{\theta}_{i j} \frac{\beta_{i j} \phi_{i j}}{\underline{\alpha}_{i j} p_{i}^{2}}
$$

However, we can demonstrate that in this case, the basis differential will also be non-negative. A proof can be constructed as follows. Observe that for the Lagrangian (22) to reach its minimum at the optimal solution, it is necessary that its second derivative with respect to any line flow must 
be non-negative. That second derivative equals

$$
\frac{\partial^{2} L}{\partial \phi_{i j}^{2}}=-\mu_{i j} \operatorname{sign}\left(\phi_{i j}\right)
$$

and therefore, whenever the flow is positive, i.e., $\phi_{i j}>0$, the corresponding dual value must be non-positive, i.e., $\mu_{i j} \leq 0$, and vise-versa. Observe that when $\underline{\alpha}_{i j}=1$, the optimality condition (23e) results in

$$
2 \mu_{i j} p_{i}^{2}=-2 m\left(\underline{\varepsilon}_{i j} \lambda_{i}+\left(1-\underline{\varepsilon}_{i j}\right) \lambda_{i}^{e}\right) \underline{\eta}_{i j} \phi_{i j}-p_{i} \underline{\xi}_{i j}^{\max }+\underline{\theta}_{i j}
$$

which, because $-\mu_{i j} \leq 0$, yields

$$
\underline{\theta}_{i j} \leq 2 m\left(\underline{\varepsilon}_{i j} \lambda_{i}+\left(1-\underline{\varepsilon}_{i j}\right) \lambda_{i}^{e}\right) \underline{\eta}_{i j} \phi_{i j}+p_{i} \underline{\xi}_{i j}^{\max }
$$

Substituting this into the expression for the basis differential equation (33) when $\underline{\alpha}_{i j}=1$, and therefore $\underline{\gamma}_{i j}=0$, yields

$$
\begin{aligned}
& \lambda_{j}-\lambda_{i}= 2 m \underline{\eta}_{i j}\left(\underline{\varepsilon}_{i j} \lambda_{i}+\lambda_{i}^{e}\left(1-\underline{\varepsilon}_{i j}\right)\right) \frac{\beta_{i j} \phi_{i j}^{2}}{p_{i}^{2}} \\
& \quad-\underline{\theta}_{i j} \frac{\beta_{i j} \phi_{i j}}{p_{i}^{2}}+\underline{\xi}_{i j}^{\max } \frac{\beta_{i j} \phi_{i j}}{p_{i}} \\
&= \frac{\beta_{i j} \phi_{i j}}{p_{i}^{2}}\left(2 m \underline{\eta}_{i j}\left(\underline{\varepsilon}_{i j} \lambda_{i}+\lambda_{i}^{e}\left(1-\underline{\varepsilon}_{i j}\right)\right) \phi_{i j}+\underline{\xi}_{i j}^{\max } p_{i}-\underline{\theta}_{i j}\right) \\
& \geq 0
\end{aligned}
$$

Note that the above discussion was applied to the case of positive flow, i.e. $\phi_{i j}>0$. Using a similar approach, it can be shown that when $\phi_{i j}<0$, the basis differential is

$$
-\left(\lambda_{j}-\lambda_{i}\right)=\bar{\delta}_{i j}+\bar{\Delta}_{i j}^{c}+\bar{\Delta}_{i j}^{p}
$$

where $\bar{\delta}_{i j}, \bar{\Delta}_{i j}^{c}$, and $\bar{\Delta}_{i j}^{p}$ are the components related to compression, capacity congestion, and pressure congestion. These components are given by

$$
\begin{aligned}
\bar{\delta}_{i j} & =\left(\bar{\varepsilon}_{i j} \lambda_{i}+\lambda_{i}^{e}\left(1-\bar{\varepsilon}_{i j}\right)\right) \bar{M}_{i j}-\bar{\theta}_{i j} \frac{\beta_{i j} \phi_{i j}}{\bar{\alpha}_{i j} p_{j}^{2}}, \\
\bar{\Delta}_{i j}^{c} & =\bar{\gamma}_{i j} \bar{M}_{i j}, \\
\bar{\Delta}_{i j}^{p} & =\bar{\xi}_{i j}^{\max } \frac{\beta_{i j} \phi_{i j}}{\bar{\alpha}_{i j} p_{j}}, \\
\bar{M}_{i j} & =\bar{\eta}_{i j}\left(\bar{\alpha}_{i j}^{2 m}-1\right)+2 m \bar{\eta}_{i j} \bar{\alpha}_{i j}^{2 m} \frac{\beta_{i j} \phi_{i j}^{2}}{\left(\bar{\alpha}_{i j} p_{j}\right)^{2}},
\end{aligned}
$$

In the following section, we apply the results of this analysis to establish revenue adequacy of the pricing mechanism.

\section{Revenue AdeQuacy}

Revenue adequacy is an important requirement for the price formation mechanism to assure that off-takers' pay- ments collected by the market administrator are sufficient to cover suppliers' receipts as well as transportation costs that include gas compressor operation. The cash balance retained by the market administrator is set by

$$
\begin{aligned}
R= & \sum_{k \in \mathcal{G}} \lambda_{j(k)}\left(d_{k}-s_{k}\right) \\
& -\sum_{j \in \mathcal{V}} \sum_{i \in \partial_{+} j} \lambda_{j}^{e}\left(1-\bar{\varepsilon}_{i j}\right) \bar{\eta}_{i j}\left|\phi_{i j}\right|\left(\left(\bar{\alpha}_{i j}^{2 m}\right)-1\right) \\
& -\sum_{j \in \mathcal{V}} \sum_{k \in \partial_{-} j} \lambda_{j}^{e}\left(1-\underline{\varepsilon}_{j k}\right) \underline{\eta}_{j k}\left|\phi_{j k}\right|\left(\left(\underline{\alpha}_{j k}^{2 m}\right)-1\right)
\end{aligned}
$$

Because we consider a single price auction mechanism, all offtakers connected to the same node pay the same price and all suppliers connected to the same node receive the same price. The first term in the equation above represents the difference between off-takers' payments and suppliers' receipts, and the second two terms represent payments for electric energy used by electrically powered compressors. As noted previously, compression can occur only at one end of any given pipe. Using the flow balance equation, the cash balance formula can be written

$$
R=\sum_{j \in \mathcal{V}} \sum_{i \in \partial_{+} j} \lambda_{j} \phi_{i j}-\sum_{j \in \mathcal{V}} \sum_{k \in \partial_{-} j} \lambda_{j} \phi_{j k}-C_{\text {comp }},
$$

where $C_{\text {comp }}$ denotes the total cost of compression. Note that in the first line of the above expression every edge appears twice, once with a plus sign and the price at the receiving node, and once with a negative sign and the price at the sending node. Thus the revenue can be written as

$$
R=\sum_{(i, j) \in \mathcal{E}}\left(\lambda_{j}-\lambda_{i}\right) \phi_{i j}-C_{\text {comp }} .
$$

The total cost of compression $C_{\text {comp }}$ is given by

$$
\begin{aligned}
C_{\text {comp }}= & \sum_{j \in \mathcal{V}} \sum_{i \in \partial_{+} j} \lambda_{j} \bar{\varepsilon}_{i j} \bar{\eta}_{i j}\left|\phi_{i j}\right|\left(\left(\bar{\alpha}_{i j}^{2 m}\right)-1\right) \\
& +\sum_{j \in \mathcal{V}} \sum_{k \in \partial_{-} j} \lambda_{j} \underline{\varepsilon}_{j k} \underline{\eta}_{j k}\left|\phi_{j k}\right|\left(\left(\underline{\alpha}_{j k}^{2 m}\right)-1\right) \\
& +\sum_{j \in \mathcal{V}} \sum_{i \in \partial_{+} j} \lambda_{j}^{e}\left(1-\bar{\varepsilon}_{i j}\right) \bar{\eta}_{i j}\left|\phi_{i j}\right|\left(\left(\bar{\alpha}_{i j}^{2 m}\right)-1\right) \\
& +\sum_{j \in \mathcal{V}} \sum_{k \in \partial_{-} j} \lambda_{j}^{e}\left(1-\underline{\varepsilon}_{j k}\right) \underline{\eta}_{j k}\left|\phi_{j k}\right|\left(\left(\underline{\alpha}_{j k}^{2 m}\right)-1\right) .
\end{aligned}
$$

By substituting the basis differential formulas (33) and (44) into equation (51), we can see that all compression cost terms cancel out and the resulting cash balance is given by (53). As demonstrated before for each branch, if the compression in the branch is active, the contribution of

$$
\begin{aligned}
& R=\sum_{\substack{(i, j) \in \mathcal{E}: \\
\phi_{i j} \geq 0}} \frac{\beta_{i j} \phi_{i j}^{2}}{\left(\underline{\alpha}_{i j} p_{i}\right)^{2}}\left(2 m \underline{\eta}_{i j}\left(\underline{\alpha}_{i j}^{2 m-1}\left(\underline{\varepsilon}_{i j} \lambda_{i}+\lambda_{i}^{e}\left(1-\underline{\varepsilon}_{i j}\right)\right)-\underline{\theta}_{i j}\right)+\sum_{\substack{(i, j) \in \mathcal{E}: \\
\phi_{i j}<0}} \frac{\beta_{i j} \phi_{i j}^{2}}{\left(\bar{\alpha}_{i j} p_{j}\right)^{2}}\left(2 m \bar{\eta}_{i j}\left(\bar{\alpha}_{i j}^{2 m-1}\left(\bar{\varepsilon}_{i j} \lambda_{j}+\lambda_{j}^{e}\left(1-\bar{\varepsilon}_{i j}\right)\right)-\bar{\theta}_{i j}\right)\right.\right. \\
& \quad+\sum_{(i, j) \in \mathcal{E}: \phi_{i j} \geq 0} \underline{\gamma}_{i j} \underline{M}_{i j}+\sum_{(i, j) \in \mathcal{E}: \phi_{i j}<0} \bar{\gamma}_{i j} \bar{M}_{i j}+\sum_{(i, j) \in \mathcal{E}: \phi_{i j} \geq 0} \underline{\xi}_{i j}^{\max } \frac{\beta_{i j} \phi_{i j}}{\underline{\alpha}_{i j} p_{i}}-\sum_{(i, j) \in \mathcal{E}: \phi_{i j}<0} \bar{\xi}_{i j}^{\max } \frac{\beta_{i j} \phi_{i j}}{\bar{\alpha}_{i j} p_{j}}
\end{aligned}
$$




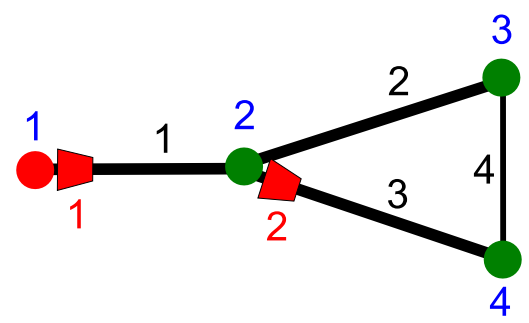

Figure 2: Simple 4-node example network.

that branch to the cash balance is strictly positive. In the absence of the compression, the contribution of the branch to the cash balance is non-negative. This proves the revenue adequacy of the locational pricing mechanism. As noted earlier, gas compressor operation costs may include the costs of gas or electricity used to drive the units. These costs are accounted for in (49) through payments to gas suppliers in the case of gas-powered compressors, and included explicitly for electrically powered compressors.

\section{Computational ExAmples}

Consider first a computational study on a simple pipeline system that connects a single supplier with three offtakers, as illustrated in Figure 2. There are two compressors that are used to propel gas, and the system has a total length of 290 miles. The system parameters are specified in Table I using standard units. The gas equation of state used is the ideal gas law with wave speed $a=1240 \mathrm{ft} / \mathrm{s}$. We ignore compression energy in this example for now in order to focus purely on congestion pricing. This can be done by setting the burn rate parameters $\eta$ to zero in equations (21a) and (21b), while keeping a positive value of $\eta$ in equation (21f). The optimization problem (21) for this case study has 12 variables, and requires less than a second to solve using an interior point method on a commodity computer [29].

Table I: Model 4 Parameter Data.

\begin{tabular}{|l|cc|cccc|cc|}
\hline \multicolumn{10}{|c|}{ Node Parameter Data } \\
\hline \hline $\begin{array}{l}\text { Node } \\
\text { id \# }\end{array}$ & $p_{j}^{\min }$ & \multicolumn{2}{|c|}{$p_{j}^{\max }$} & $d_{j}^{\min }$ & $d_{j}^{\max }$ & $s_{j}^{\min }$ & $s_{j}^{\max }$ & \multicolumn{2}{|c|}{$\begin{array}{c}\text { psmscfd } \\
\text { mmscfd }\end{array}$} & \multicolumn{7}{c|}{ offer } & bid \\
\hline 1 & 600 & 1000 & 0 & 0 & 400 & 2000 & $\$ 1$ & - \\
2 & 300 & 1000 & 0 & 800 & 0 & 0 & - & $\$ 2$ \\
3 & 300 & 1000 & 0 & 600 & 0 & 0 & - & $\$ 4$ \\
4 & 300 & 1000 & 0 & 2000 & 0 & 0 & - & $\$ 3$ \\
\hline
\end{tabular}

\begin{tabular}{l|ccccc|}
\hline \multicolumn{7}{|c|}{ Pipe Parameter Data } \\
\hline \hline Pipe & From node & To node & $D_{i j}$ & $L_{i j}$ & $f_{i j}$ \\
id \# & $i$ & $j$ & inches & miles & \\
\hline 1 & 1 & 2 & 36 & 50 & 0.01 \\
2 & 2 & 3 & 36 & 80 & 0.01 \\
3 & 2 & 4 & 36 & 80 & 0.01 \\
4 & 3 & 4 & 25 & 80 & 0.01 \\
\hline
\end{tabular}

\begin{tabular}{|l|ccc|}
\hline \multicolumn{4}{c|}{ Compressor Parameter Data } \\
\hline \hline Compressor & $\begin{array}{c}\text { Location node } \\
\text { node id \# }\end{array}$ & $\begin{array}{c}\text { To edge } \\
\text { edge id \# }\end{array}$ & $\begin{array}{c}E_{i j}^{\max } \\
\text { horsepower }\end{array}$ \\
\hline 1 & 1 & 1 & 6000 \\
2 & 2 & 3 & 4000 \\
\hline
\end{tabular}

The results of the optimization case study are shown in Table II. Examining the marginal prices $\lambda_{j}$, we see that the price at node 2 is lowest, while the price at node 3 is highest, and node 4 is the marginal offtaker. We find that the price downstream of node 2 is high enough for no deliveries to be made there for this particular parameter set. Indeed, capacity is maximally utilized, because the discharge pressure of compressor 1 is set to the MAOP of 1000 psia. This is the binding constraint related to compressor 1. Next, observe that compressor 2 is operating at its horsepower bound. Finally, the pressure at node 3 is at the minimum, because the offtake at that location is maximized.

Because the compression costs were omitted in the optimization, and the compression ratios satisfy $\underline{\alpha}_{i j}>1$, the compression component $\underline{\delta}_{i j}$ will not appear in the basis differential equation (33). However, it is a straightforward exercise to verify the congestion components corresponding to compressor power and pressure limits given by $\underline{\Delta}_{i j}^{c}$ and $\Delta_{i j}^{p}$. Further investigation of even this simple example can provide insight into the interaction of physical, economic, and engineering aspects of gas pipeline systems.

Table II: Model 4 Solution Data.

\begin{tabular}{|l|cc|c|}
\hline \multicolumn{4}{|c|}{ Node Solution Data } \\
\hline \hline id \# & $\begin{array}{c}\text { Consumption } d_{j} \\
\text { mmscfd }\end{array}$ & Supply $s_{j}$ & $\begin{array}{c}\text { Marginal Price } \lambda_{j} \\
\$ / \text { mmscfd }\end{array}$ \\
\hline 1 & - & 1475.9 & - \\
2 & 0 & - & 2.6481 \\
3 & 600 & - & 3.1650 \\
4 & 875.89 & - & 3.0000 \\
\hline
\end{tabular}

\begin{tabular}{|l|cccccc|}
\hline \multicolumn{7}{|c|}{ Pipe Solution Data } \\
\hline \hline Pipe & From node & To node & $\underline{p}_{i j}$ & $\bar{p}_{i j}$ & $\phi_{i j}$ \\
id \# & $i$ & $j$ & \multicolumn{2}{c|}{ psia } & mmscfd \\
\hline 1 & 1 & 2 & 1000 & 474.41 & 1475.9 \\
2 & 2 & 3 & 474.41 & 300 & 487.12 \\
3 & 2 & 4 & 831.51 & 367.30 & 988.76 \\
4 & 3 & 4 & 300 & 367.30 & -112.87 \\
\hline
\end{tabular}

\begin{tabular}{|l|cccc|}
\hline \multicolumn{5}{c|}{ Compressor Solution Data } \\
\hline \hline Compressor & $\begin{array}{c}\text { Location node } \\
\text { id \# }\end{array}$ & To edge & $\underline{\alpha}_{i j}$ & $\underline{E}_{i j}$ \\
\hline 1 & 1 & 1 & 1.666 & 5256.4 \\
2 & 2 & 3 & 1.7527 & 4000.0 \\
\hline
\end{tabular}

To demonstrate scalability, we formed a case study using the Gaslib-40 network instance [30]. This system has 40 nodes, 45 pipes, and a total system length of 695 miles, with supply at nodes 1,2 , and 3 , where we set offer prices (per mmscfd) to $\$ 1, \$ 1.5$, and $\$ 2$, respectively. We set bids at $\$ 3$ per mmscfd at most offtaker locations, and set bids of $\$ 4$ at nodes $22,24,25$, and 34 , and bids of $\$ 8$ at nodes 16, 18, 20, and 31. Maximum offtakes for any consumer were set to $800 \mathrm{mmscfd}$, and this constraint was binding at nodes 16, 18, 22, and 39, while nodes 6, 20, 26, 31, 34, and 36 had lesser nonzero offtakes. Maximum power for the compressors on edges 43 and 44 were limited to 3000 and 2000 horsepower, respectively, and were binding. Minimum 

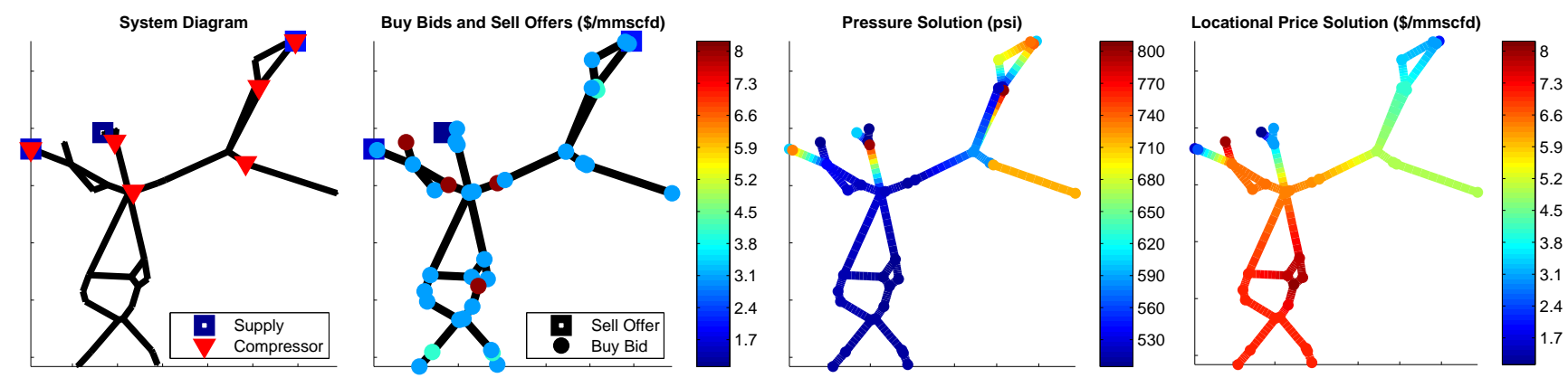

Figure 3: Solution for Gaslib-40 example. From left to right: System diagram; Supplier offers and offtaker bids; Pressure solution; Marginal price solution. High offers and capacity constraints drive price formation.

and maximum system pressures were set to 500 and 800 psi, respectively. The problem, which has 125 variables, is solved using an interior point method [29] in about 0.85 seconds. Figure 3 illustrates the results.

\section{CONCLUSIONS}

We have presented a locational marginal pricing mechanism to maximize social welfare for a gas pipeline network with steady-state flow. Optimality conditions are derived that lead to locational marginal prices for gas (gLMPs) that account for physical and engineering system constraints. These gLMPs provide price signals that quantify the cost of physical transportation of gas through the system. The pricing system is shown to be revenue adequate. A simple, interpretable computational example was explored, and a larger and more complex example was examined to demonstrate scalability of the approach to real systems.

Our focus here is on developing an economic theory of optimal pipeline network operation that can lead to methods for optimal day-ahead and intra-day flow scheduling. At these time scales, we assume that the economics of natural gas production and supply are embedded in the offer prices of consumers and suppliers. This preliminary study does not consider co-optimization of pipeline transportation and storage operations, but rather assume that the economic aspects of storage injections and withdrawals are reflected in corresponding price offers and bids.

The key theoretical instrument used in this paper is the analysis of Karush Kuhn Tucker (KKT) conditions for optimality. The steady-state pipeline optimization problem examined is non-convex, so the KKT conditions are not sufficient to prove optimality or construct solutions, nor are they used here for those purposes. Computational approaches have been shown to effectively solve large-scale pipeline optimization problems [23], [24]. The primary focus of this paper is rather on the introduction and derivation of properties of gLMPs. As necessary conditions of optimality, the KKT conditions provide a powerful instrument for studying the properties of Lagrange multipliers as those properties must necessarily hold at the optimal solution of the problem. In general, that part of the KKT theorem does not require convexity of the optimization problems. On the other hand, the KKT theorem does not by itself assert that these Lagrange multipliers could be interpreted as shadow prices for the associated constraints. The use in economic theory of certain Lagrange multipliers as prices of goods or services requires justification. The latter is typically provided by envelope theorems that address the differentiability properties of the objective function of a parameterized optimization problem [31]. Crucially, the key results of the envelope theorem do not require convexity and remain true under significantly less stringent assumptions. This has been discussed in Section IV above.

The above steady-state modeling approach is valid for coarse price formation over large-scale systems on time scales of weeks. Appropriate engineering models must be used for such economic optimization problems to suitably represent physical flows on the relevant time and space scales. Recently, regulatory changes in the United States have opened the path to physics-based gas pricing mechanisms with intra-day time granularity [10], [11], which compels further investigation [9]. This work is a step towards developing the theory and computation of dynamic price formation for intra-day flows. Extension to multi-period economic optimization that incorporates transient hydraulic models [32], [23], sensitivity analysis for larger case studies, and global optimization by adaptive partitioning mixedinteger relaxations [33] are promising future directions.

\section{ACKNOWLEDGEMENTS}

We thank Sidhant Misra, Marc Vuffray, Michael Chertkov, Scott Backhaus, Evgeniy Goldis, Pablo Ruiz, Russ Philbrick and Richard Tabors for discussions and suggestions. This work was carried out as part of Project GECO for the Advanced Research Project Agency-Energy of the U.S. Department of Energy under Award No. DE-AR0000673. Work at Los Alamos National Laboratory was conducted under the auspices of the National Nuclear Security Administration of the U.S. Department of Energy under Contract No. DEAC52-06NA25396, and was supported by the Advanced Grid Modeling Research Program in the U.S. Department of Energy Office of Electricity. 


\section{REFERENCES}

[1] C. Lyons and G. Litra, "Gas-power interdependence: Knock-on effects of the dash to gas," 2013, http://www.scottmadden.com/insight/598/gaspowerinterdependence.html.

[2] S. E. Jenkins, "Interdependency of electricity and natural gas markets in the united states: a dynamic computational model," Master's thesis, Massachusetts Institute of Technology, 2014.

[3] R. Levitan, S. Wilmer, and R. Carlson, "Pipeline to reliability: Unraveling gas and electric interdependencies across the eastern interconnection," Power and Energy Magazine, IEEE, vol. 12, no. 6, pp. 78-88, 2014.

[4] M. Chertkov, S. Backhaus, and V. Lebedev, "Cascading of fluctuations in interdependent energy infrastructures: Gas-grid coupling," Applied Energy, vol. 160, pp. 541 - 551, 2015.

[5] T. Pugh and J. P. Blackford, "Implications of greater reliance on natural gas for electric generation," 2010

[6] M. Shahidehpour and Z. Li, "White paper: Long-term electric and natural gas infrastructure requirements," Illinois Institute of Technology, Tech. Rep., 2014.

[7] P. W. MacAvoy, The natural gas market: Sixty years of regulation and deregulation. Yale University Press, 2008.

[8] R. D. Tabors, S. Englander, and R. Stoddard, "Who's on first? the coordination of gas and power scheduling," The Electricity Journal, vol. 25, no. 5, pp. 8-15, 2012.

[9] R. Carter et al., "Impact of regulatory change to coordinate gas pipelines and power systems," in PSIG Annual Meeting. Pipeline Simulation Interest Group, 2016.

[10] "Federal Energy Regulatory Commission (FERC) order \#787," 2013, www.ferc.gov/CalendarFiles/20131115164637RM13-17-000.pdf.

[11] "Federal Energy Regulatory Commission (FERC) order \#809," 2015, http://www.ferc.gov/whats-new/commmeet/2015/041615/M-1.pdf.

[12] E. Litvinov, "Design and operation of the locational marginal prices-based electricity markets," Generation, Transmission Distribution, IET, vol. 4, no. 2, pp. 315-323, February 2010.

[13] Forward Market Operations, "Energy \& Ancillary Services Market Operations, M-11 Rev. 75,” PJM, Tech. Rep., 2015.

[14] W. Pepper, B. J. Ring, E. G. Read, and S. R. Starkey, "Implementation of a scheduling and pricing model for natural gas," in Handbook of networks in power systems II. Springer, 2012, pp. 3-35.

[15] A. Bermúdez et al., "Gas transport networks: Entry-exit tariffs via least squares methodology," Energy Policy, vol. 63, pp. 252-260, 2013.

[16] A. Zlotnik, L. Roald, S. Backhaus, M. Chertkov, and A. G., "Efficient dynamic compressor optimization in natural gas transmission systems," in IEEE American Control Conference, Boston, MA, 2016, pp. 7478-7483.
[17] E. S. Menon, Gas pipeline hydraulics. CRC Press, 2005

[18] T. Koch, B. Hiller, M. Pfetsch, and L. Schewe, "Evaluating gas network capacities," 2015.

[19] R. D. Tabors and S. Adamson, "Measurement of energy market inefficiencies in the coordination of natural gas \&; power," in 47th Hawaii International Conference onSystem Sciences (HICSS). IEEE, 2014, pp. 2335-2343.

[20] Office of Pipeline and Producer Regulation and Office of Economic Policy, "Gas transportation rate design and the use of auctions to allocate capacity," Federal Energy Regulatory Commission, Tech. Rep., 1987.

[21] E. G. Read, B. J. Ring, S. R. Starkey, and W. Pepper, "An lp based market design for natural gas," in Handbook of networks in power systems II. Springer, 2012, pp. 77-113.

[22] T. W. K. Mak, P. Van Hentenryck, A. Zlotnik, H. Hijazi, and R. Bent, "Efficient dynamic compressor optimization in natural gas transmission systems," in IEEE American Control Conference, Boston, MA, 2016, pp. 7484-7491.

[23] A. Zlotnik, M. Chertkov, and S. Backhaus, "Optimal control of transient flow in natural gas networks," in 54th IEEE Conference on Decision and Control, Osaka, Japan, 2015, pp. 4563-4570.

[24] A. Zlotnik, S. Misra, M. Vuffray, and M. Chertkov, "Monotonicity of actuated flows on dissipative transport networks," European Control Conference (ECC), pp. 831-836, 2016.

[25] L. W. Davis and E. Muehlegger, "Do Americans consume too little natural gas? An empirical test of marginal cost pricing," The RAND J. of Economics, vol. 41, no. 4, pp. 791-810, 2010.

[26] D. G. Luenberger, Introduction to linear and nonlinear programming. Reading, MA: Addison-Wesley, 1973, vol. 28.

[27] P. Milgrom and I. Segal, "Envelope theorems for arbitrary choice sets," Econometrica, vol. 70, no. 2, pp. 583-601, 2002.

[28] A. D. Ioffe and V. M. Tikhomirov, Theory of Extremal Problems. North-Holland Publishing, 1979.

[29] L. T. Biegler and V. M. Zavala, "Large-scale nonlinear programming using IPOPT: An integrating framework for enterprise-wide dynamic optimization," Computers \& Chem. Engineering, vol. 33, no. 3, pp. 575-582, 2009.

[30] J. Humpola et al., "Gaslib: a library of gas network instances," 2015.

[31] L. M. Benveniste and J. A. Schenkman, "On the differentiability of the value function in dynamic models of economics," Econometrica, vol. 47, no. 3, pp. 727-732, 1979.

[32] A. J. Osiadacz and M. Chaczykowski, "Dynamic control for gas pipeline systems," Archives of Mining Sciences, vol. 61, no. 1, pp. 69-82, 2016.

[33] H. Nagarajan, M. Lu, E. Yamangil, and R. Bent, "Tightening mccormick relaxations for nonlinear programs via dynamic multivariate partitioning," in International Conference on Principles and Practice of Constraint Programming. Springer, 2016, pp. 369-387. 